\title{
Severed Actin and Microtubules with Motors Walking All Over Them: Cryo-EM Studies of Seriously Perturbed Helical Assemblies
}

\author{
Garrett Debs ${ }^{1}$, Andrew Huehn ${ }^{1}$, Michael Cha ${ }^{1}$, Xueqi Liu ${ }^{1}$, William A. Elam ${ }^{1,2}$, Wenxiang Cao ${ }^{1}$, Enrique \\ De La Cruz ${ }^{1}$ and Charles V. Sindelar ${ }^{*}$ \\ 1. Department of Molecular Biophysics and Biochemistry, Yale University, New Haven, CT, USA. \\ 2. Present address: C4 Therapeutics, Cambridge, MA, USA. \\ * Corresponding author: chuck.sindelar@yale.edu
}

The symmetry of helical biofilaments can be advantageous for structure determination by cryo-EM because extra averaging can be performed, using known geometric relationships between filament subunits. However, filaments become much harder to analyze when they deviate significantly from strict helical symmetry, which can happen when there is significant structural and/or compositional heterogeneity of the helical subunits. Here, we present new findings on two processes where helical symmetry breaking has special functional relevance: (1) the severing of actin filaments by its binding partner, cofilin; and (2) microtubules irregularly covered by kinesin motor proteins. By developing specialized methods for analyzing cryo-EM images of filament assemblies with broken symmetry, we obtained 3D maps of previously inaccessible structural states of these samples, yielding new functional insights into biological behaviors of these assemblies.

Mechanism of actin severing by cofilin. In our recent study of actin severing induced by cofilin [1], we used a variation of focused classification [2], specially adapted by us for helical filaments, to show that cofilin binding induces abrupt changes in actin filament helical geometry. This furnished a key piece of evidence in support of a 'phase boundary'-type severing mechanism [3]. We have now extended this work to obtain a sub-nanometer resolution 3D reconstruction of the cofilin-induced 'fracture zone' by identifying and aligning a rare population of boundary-containing filament segments ( $~ 800$ particles) from 3000 images of actin filaments decorated by sub-stoichiometric ratios of cofilin (Fig. 1A). Further application of our classification approach revealed additional intermediates in the severing pathway (Fig. 1B), which we are currently integrating into a model for how cooperative binding of cofilin weakens the actin filament at boundaries between bare and decorated regions, leading to severing.

Structural basis of motility in kinesin molecular motor proteins. Adapting our focused classification method to microtubules, we developed a 'patch' method (Fig. 2A) that allowed us to address non-helical deformations of the microtubule wall, and to structurally classify a minority of sites occupied by kinesin molecular motors. Using this approach, we obtained a structure of the microtubule at $2.9 \AA$ resolution (Fig. 2B) and captured a series of key structural intermediates describing a forward step by the dimeric form of kinesin (Fig. 2C). We also obtained a near-atomic resolution structure of a single 'foot' of the kinesin-1 motor bound to the microtubule after it lands and releases ADP (Fig. 2D), providing the first direct validation for structure models of this state presented two years ago by our group and others [4, 5]. Finally, we discovered a 'crinkling' behavior at the microtubule seam featuring an unexpectedly large rotation $\left(\sim 15^{\circ}\right)$ between adjacent protofilaments (Fig. 3). These results pave the way for further studies of kinesin, microtubules and other microtubule assemblies, potentially approaching atomic resolution where chemical details could be directly observed. 
References:

[1] A. Huehn et al., J Biol Chem 293 (2018), p. 5377.

[2] XC Bai et al., Elife, 2015. 4.

[3] E De La Cruz, Biophysical Reviews 1 (2009), p. 51.

[4] L Cao et al., Nat Commun 5 (2014), p. 5364.

[5] Z Shang et al., Elife, 3 (2014), p. e04686.
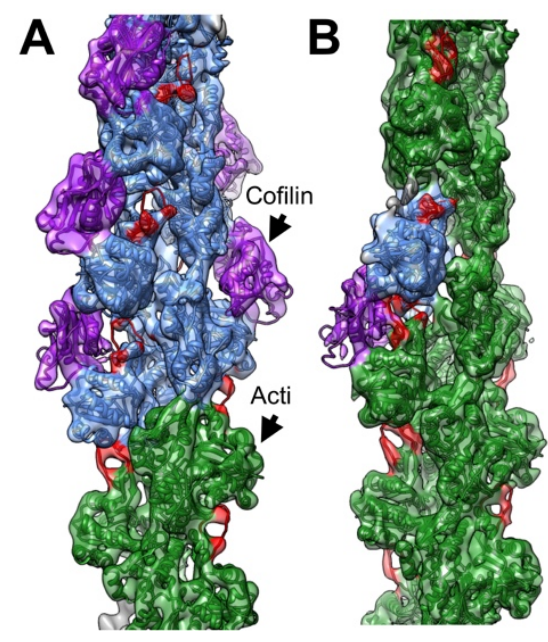

Figure 1. Intermediates in the actin severing pathway by cofilin. A, Sub-nanometer cryo-EM map and fitted model of a boundary between cofilin-decorated (top) and bare (bottom) actin. $\mathrm{B}$, Bare actin filament with a single bound cofilin subunit.
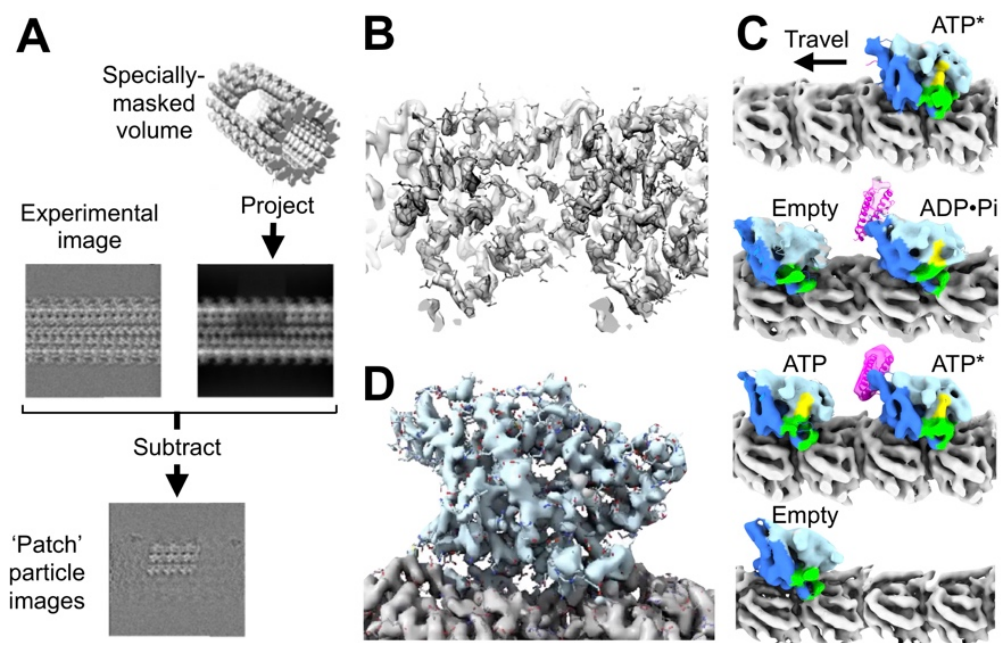

Figure 2. Reconstructed microtubule complexes using a 'patch' method. A, Patch method schematic. B, Close-up of a $2.9 \AA$ microtubule reconstruction. $\mathrm{C}$, Sequence of states representing dimeric kinesin stepping along a microtuble. $\mathrm{D}$, near-atomic reconstruction of a kinesin head after ADP release.
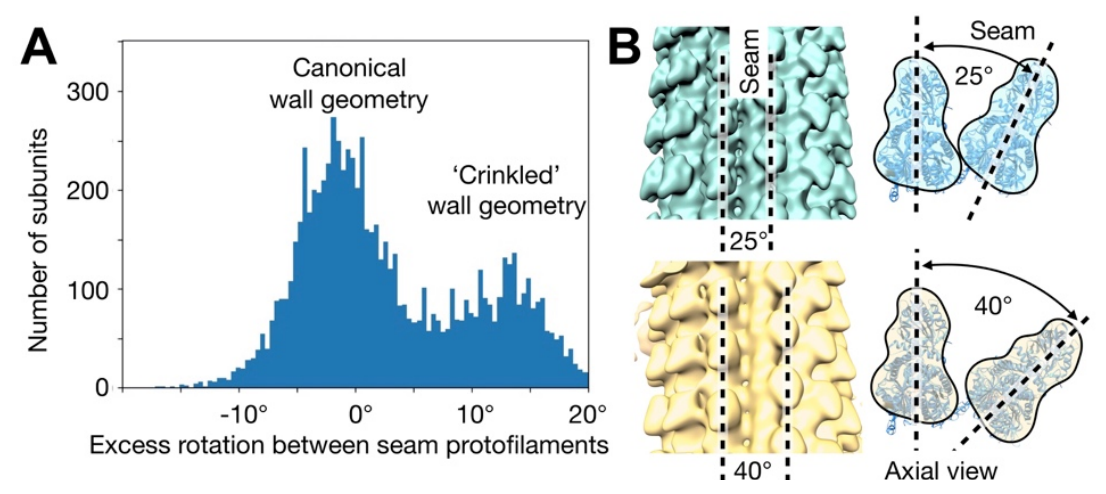

Figure 3. Geometric distortions of the microtubule seam discovered by 'patch' analysis. A, Histogram of seam distortions estimated using single-protofilament 'patches'. B, 3D reconstructions of the canonical (top) and 'wrinkled' (bottom) seam 